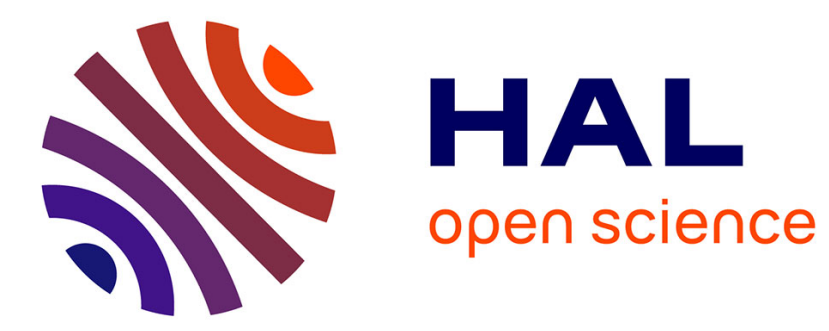

\title{
Histamine quantification in human plasma using High Resolution Accurate Mass LC-MS technology
}

Mathieu Laurichesse, Thomas Gicquel, Caroline Moreau, Olivier Tribut, Karin Tarte, Isabelle Morel, Claude Bendavid, Patricia Amé-Thomas

\section{- To cite this version:}

Mathieu Laurichesse, Thomas Gicquel, Caroline Moreau, Olivier Tribut, Karin Tarte, et al.. Histamine quantification in human plasma using High Resolution Accurate Mass LC-MS technology. Clinical Biochemistry, 2016, 49 (1-2), pp.111-116. 10.1016/j.clinbiochem.2015.08.012 . hal-01187144

HAL Id: hal-01187144

https://hal-univ-rennes1.archives-ouvertes.fr/hal-01187144

Submitted on 22 Sep 2015

HAL is a multi-disciplinary open access archive for the deposit and dissemination of scientific research documents, whether they are published or not. The documents may come from teaching and research institutions in France or abroad, or from public or private research centers.
L'archive ouverte pluridisciplinaire HAL, est destinée au dépôt et à la diffusion de documents scientifiques de niveau recherche, publiés ou non, émanant des établissements d'enseignement et de recherche français ou étrangers, des laboratoires publics ou privés. 


\section{Histamine quantification in human plasma using High Resolution Accurate Mass LC-MS technology}

Mathieu LAURICHESSE ${ }^{1,2}$, Thomas GICQUEL ${ }^{3,4}$ Caroline MOREAU $^{2,5}$, Olivier TRIBUT ${ }^{6}$, Karin TARTE ${ }^{1,5,7}$, Isabelle MOREL ${ }^{3,4}$, Claude BENDAVID ${ }^{2,4}$, Patricia AME-THOMAS ${ }^{1,5,7}$

1- CHU Rennes, Laboratoire d'Immunologie, Thérapie Cellulaire et Hématopoïèse, F35033 Rennes, France

2- CHU Rennes, Laboratoire de Biochimie, F-35033 Rennes, France

3- CHU Rennes, Laboratoire de Toxicologie Biologique et Médico-Légale, F-35033 Rennes, France

4-Université de Rennes 1, Faculté de Pharmacie, F-35043 Rennes, France

5- Université de Rennes 1, Faculté de Médecine, F-35043 Rennes, France

6-CHU Rennes, UF Biomarqueurs, F-35033 Rennes, France

7- INSERM Unité Mixte de Recherche 917, F-35043 Rennes, France

Correspondence:

Mathieu LAURICHESSE Laboratoire d'immunologie CHU Pontchaillou

2 Avenue du Pr Léon Bernard 35033 Rennes, France.

E-mail: m_laurichesse@hotmail.com

Phone number: +33676216897

Patricia AME-THOMAS Laboratoire d'immunologie CHU Pontchaillou

2 Avenue du Pr Léon Bernard 35033 Rennes, France.

E-mail: patricia.ame@univ-rennes1.fr

Phone number: +33223234827

3624 Words, 3 Figures, 3 Tables, and 27 References 


\section{ABSTRACT}

Background: Histamine (HA) is a small amine playing an important role in anaphylactic reactions. In order to identify and quantify HA in plasma matrix, different methods have been developed but present several disadvantages. Here, we developed an alternative method using liquid chromatography coupled with an ultra-high resolution and accurate mass instrument, Q Exactive $^{\mathrm{TM}}$ (Thermofisher) (LCHRMS).

Methods: The method includes a protein precipitation of plasma samples spiked with HA-d4 as internal standard (IS). LC separation was performed on a C18 Accucore column (100*2.1 $\mathrm{mm}, 2.6 \mu \mathrm{m})$ using a mobile phase containing nonafluoropentanoic acid $(3 \mathrm{nM})$ and acetonitrile with $0.1 \%(\mathrm{v} / \mathrm{v})$ formic acid on gradient mode. Separation of analytes was obtained within $10 \mathrm{~min}$. Analysis was performed from full scan mode and targeted MS2 mode using a 5 ppm mass window. Ion transition monitored for targeted MS2 mode were $112.0869>95.0607 \mathrm{~m} / \mathrm{z}$ for HA and $116.1120>99.0855 \mathrm{~m} / \mathrm{z}$ for HAd4. Calibration curves were obtained by adding standard calibration dilution at 1 to $180 \mathrm{nM}$ in TrisBSA.

Results: Elution of HA and IS occurred at $4.1 \mathrm{~min}$. The method was validated over a range of concentrations from $1 \mathrm{nM}$ to $100 \mathrm{nM}$. The intra- and inter-run precisions were $<15 \%$ for quality controls. Human plasma samples from 30 patients were analysed by LCHRMS, and the results were highly correlated with those obtained using the gold standard Radioimmunoassay (RIA) method.

Conclusion: Overall, we demonstrate here that LCHRMS is a sensitive method for histamine quantification in biological human plasmas, suitable for routinely use in medical laboratories. In addition, LCHRMS is less time-consuming than RIA, avoid the use of radioactivity, and could then be considered as an alternative quantitative method. 
Keywords: Histamine, Accurate Mass, LC-High Resolution-MS, anaphylactic reaction

Abbreviations: HA: Histamine

LCHRMS: Liquid Chromatography - High Resolution - Mass Spectrometry NFPA: Nonafluoropentanoic acid

RIA: Radioimmunoassay 


\section{Introduction}

Histamine (HA) $\left(\mathrm{M}=111.1451\right.$ g.mol $\left.{ }^{-1}\right)$ had been discovered in 1910 by Dale and Laidlaw [1]. HA is a small organic nitrogenous molecule known to play an important role in allergic reactions. Indeed, mast cells and basophils are specifically prone to synthetize HA, and to secrete it after IgE-dependent activation and degranulation during type I hypersensitivity responses. Binding of HA to its four different receptors [histamine 1 receptor (H1R), H2R, $\mathrm{H} 3 \mathrm{R}$ and H4R] present on various cells in different tissues has been associated with clinical symptoms and metabolic functions. It is now well documented that binding of HA to H1R and/or H2R leads to vasodilatation, blood pressure diminution, mucus secretion, or even gastric acid secretion. Due to H3R ligation, HA plays a role in various neurological processes such as food intake or memory, and in neuropathology [2]. As described by Rosa and Fantozzi [3], HA could play an important role in neurogenic inflammation, particularly in nociceptive pain but also in neurogenic inflammation of skin, airways and bladder. Finally, many findings confer to HA a physiologic role during pregnancy [4][5].

During type I hypersensitivity responses, only 2 biological markers seem relevant and are currently quantified in routine laboratories to validate the allergic reaction: HA and tryptase. HA release could be measured in plasma, but also in other matrix such as urine [6], cerebrospinal fluid [7], or even cell culture supernatant [8]. Due to HA low concentrations in these different fluids even after an important release, sensitive quantification methods are needed. Various analytical methods have already been developed to identify and quantify HA in biological samples: liquid chromatography (LC) [9][10], gas chromatography (GC) [11][12], capillary electrophoresis (CE) [13] coupled with different detection modes. Derivatization was usually used to improve the sensitivity, but was time-consuming, and increased the risk of low analyte recovery. Other non-separative methods like radioimmunoassay (RIA) [14][15] or enzyme immunoassays (EIA) [16] are currently the most common methods used in medical laboratories. RIA is yet considered as the gold standard method. However, RIA is highly time-consuming, and protective measures against radiations and exposure monitoring of the technicians due to use of radioactivity are necessary. In addition, cross reactivity with 3methylhistamine, the main metabolite of HA in vivo, is still an issue for RIA and EIA methods [17].

The definition of new methods combining high resolution and high throughput analyses could be useful. To our knowledge, no method using Liquid Chromatography - High Resolution Mass Spectrometry (LCHRMS) to quantify HA in plasma samples has been yet developed and validated for medical laboratory application. Herein, we describe a validated method using liquid chromatography coupled with an ultra-high resolution and accurate mass 
instrument (Q Exactive ${ }^{\mathrm{TM}}$ ) for HA quantification in plasma from patients suspected to develop an anaphylactic reaction. This method was compared to the RIA gold standard method, and was found to be routinely usable in medical laboratories.

\section{Experimental}

\subsection{Chemicals and reagents}

Histamine (HA) $\left(\mathrm{C}_{5} \mathrm{H}_{9} \mathrm{~N}_{3}\right)$, nonafluoropentanoic acid (NFPA), sulfosalycylic acid (SSA), trifluoroacetic acid (TFA), Tris, $\mathrm{NaCl}, \mathrm{KCl}, \mathrm{CaCl}_{2}, \mathrm{MgCl}_{2}$ and Bovine Serum Albumin (BSA) were obtained from Sigma-Aldrich (St Quentin Fallavier, France). Acetonitrile (ACN) was obtained from Thermo Fisher (Elancourt, France). Deuterated Histamine (HAd4) (Histamine$\alpha, \alpha, \beta, \beta-\mathrm{d} 4$ ), the internal standard (IS), was obtained from $\mathrm{C} / \mathrm{D} / \mathrm{N}$ Isotopes (Quebec, Canada). TrisBSA solution was prepared as follow: $3.02859 \mathrm{~g}$ of Tris, $7.0128 \mathrm{~g} \mathrm{NaCl}, 0.3728 \mathrm{~g} \mathrm{KCl}$, $0.0445 \mathrm{~g} \mathrm{CaCl} 2,0.1016 \mathrm{~g} \mathrm{MgCl} 2$, and $0.3 \mathrm{~g} \mathrm{BSA}$ were dissolved in 1 litre of water. All chemicals, reagents and solvents were of LC/MS quality grade.

\subsection{Instrumentation}

Analyses were performed on a Thermo Scientific Q Exactive ${ }^{\mathrm{TM}}$ (San Jose, USA) mass spectrometer including an Accela pump (Thermo Scientific, San Jose, USA). A heated electrospray ionisation-II (HESI-II) ion source was used for the ionization of target compounds. Data acquisition, peak integration and calibration were performed using Xcalibur $^{\circledR} 2.1$ software (ThermoScientific, San Jose, CA, USA).

\subsection{LC conditions}

LC separation was performed using a C18 Accucore column (100 mm*2.1, $2.6 \mu \mathrm{m})$ (ThermoScientific, San Jose, CA, USA) thermostated at $40^{\circ} \mathrm{C}$. The mobile phases used were NFPA (3 mmol.L ${ }^{-1}$; solvent A) and ACN with $0.1 \%(\mathrm{v} / \mathrm{v})$ formic acid (solvent B) in a mobile phase as described in Table 1. All prepared samples were kept at $15^{\circ} \mathrm{C}$ in the auto-sampler until injection of $20 \mu \mathrm{L}$ into the LCHRMS system (full loop). Sampling needle was washed with a flush $(75 \% \mathrm{ACN}, 25 \%$ water containing $0.1 \%$ TFA).

\subsection{MS conditions}


The source HESI-II was set up as follow: probe at $300^{\circ} \mathrm{C}$ was operated in positive electrospray ionization mode with spray voltage of $3 \mathrm{kV}$, sheath gas and auxiliary gas $\left(\mathrm{N}_{2}\right)$ were pressurized at 40 psi and 10 arbitrary units respectively, capillary temperature was set at $300^{\circ} \mathrm{C}$ and source lens at $60 \mathrm{~V}$. Data were acquired simultaneously in full scan with highresolution acquisition and targeted MS2 modes. In full scan acquisition, resolution was set at 70000 FWHM. The C-trap capacity was set at $10^{6}$ charges and the maximum injection time at $200 \mathrm{~ms}$. The mass range was set from 100 to $123 \mathrm{~m} / \mathrm{z}$. In targeted MS2 mode, resolution was set at 17500 FWHM. The C-trap capacity was set at $5.10^{5}$ charges and the maximum injection time at $100 \mathrm{~ms}$. The isolation window was set at $1.0 \mathrm{~m} / \mathrm{z}$ and the normalized collision energy at $45 \%$. In full scan mode, the exact mass of each protonated species (112.0869 for HA and 116.1120 for HAd4) was extracted for quantification, using a 5 ppm extraction window. In addition, to be sure of the specificity and selectivity of the used quantifier mass, one ion transition was monitored per compound in fragmentation mode (HA: $112.0869>95.0607 \mathrm{~m} / \mathrm{z}$; and HAd4: $116.1120>99.0855 \mathrm{~m} / \mathrm{z}$ ).

\subsection{Standard solutions for LCHRMS}

Stock solutions of HA $(100 \mu \mathrm{M})$ and the IS HAd4 $(10 \mu \mathrm{M})$ were prepared by dissolving each accurately weighed compound in a known volume of de-ionized water, and were stored at $80^{\circ} \mathrm{C}$. HA calibration standard solutions $(1,5,20,60,120$ and $180 \mathrm{nM})$ were daily prepared by dilution of the stock solution of HA with de-ionized water. Working IS solution (25 nM) was prepared by dilution of the stock IS solution in de-ionized water.

\subsection{Quality Controls preparation}

Quality control (QC) samples were prepared at three concentrations of HA (3, 30 and $150 \mathrm{nM})$ by fortifying TrisBSA solution, and stored at $-80^{\circ} \mathrm{C}$. $100 \mu \mathrm{L}$ of QC was added to $250 \mu \mathrm{L}$ of deionized water and $100 \mu \mathrm{L}$ of IS. SSA $(50 \mu \mathrm{L}$ at $20 \% \mathrm{v} / \mathrm{v})$ was added in each tube for protein precipitation before vortexing for 30 seconds. After centrifugation at $3000 \mathrm{~g}$ for 10 minutes at $4^{\circ} \mathrm{C}, 200 \mu \mathrm{L}$ of supernatant were transferred to an auto-sample vial.

\subsection{Calibration and linearity}

$100 \mu \mathrm{L}$ of each calibration standard solution was added to $100 \mu \mathrm{L}$ of TrisBSA, $150 \mu \mathrm{L}$ of deionized water, $100 \mu \mathrm{L}$ of IS, and $50 \mu \mathrm{L}$ of SSA at $20 \%(\mathrm{v} / \mathrm{v})$. After extraction, the six calibration points were analysed. Standard curves corresponded to peak area ratios of HA to IS using 
weighted linear least-squares regression $(1 / \mathrm{x})$, and coefficients of determination $\left(\mathrm{r}^{2}\right)$ were calculated. Mean and standard deviation (SD) were calculated from six calibration curves run each day, and precision was represented by the coefficient of variation $(\mathrm{CV}=100 * \mathrm{SD} /$ mean $)$ between each calibration point run 6 times.

\subsection{Recovery, process efficiency and matrix effect}

Recovery (RE), matrix effects (MEs), and process efficiency (PE) were performed in QC samples at 3 concentrations $(3,30$ and $150 \mathrm{nM}$ ) in analogy to the simplified approach described by [18] et al . Briefly, RE was calculated by comparing average peak areas of TrisBSA fortified by the same concentrations of QC samples, before or after the extraction procedure. PE was determined by comparing average peak areas of TrisBSA fortified prior to extraction with peak areas of samples at the same nominal concentrations prepared in water (neats). MEs were calculated as follows: $(100 *$ mean peak area of fortified TrisBSA after extraction / mean peak area of neats) -100 .

\subsection{Precision and accuracy}

The repeatability (intraday precision) was evaluated by analysing 6 different samples of each QC concentration $(3,30,150 \mathrm{nM})$ in the same day. For reproducibility (interday precision), 10 different day analyses were assessed for the 3 QC samples. The calculated values were based on a daily calibration curve. Precision was calculated by using the coefficient of variation (CV $\left.\%=(\mathrm{SD} / \mathrm{M})^{*} 100\right)$; where $\mathrm{M}$ is the mean of the experimentally determined concentrations and SD the standard deviation of $M$ and the bias was used to express accuracy [19]. The assay acceptance criterion for each concentration was $\pm 15 \%$ deviation of the nominal concentration, except for the lower limit of quantification (LLOQ) for which a deviation of $\pm 20 \%$ was accepted.

\subsection{Stability}

Long-term stability of stock solutions was evaluated by comparing stored and fresh stock solutions. Long-term stability of HA was checked by measuring QC samples (3, 30, and $150 \mathrm{nM}$ ) stored at $-80^{\circ} \mathrm{C}$ during 6 months using a daily calibration curve. The stability of HA after extraction was tested on the same extract injected at the beginning and at the end of an analytical run of 12 hours. This stability was checked at 3 levels (3, 30 and $150 \mathrm{nM})$ of spiked plasma. 


\subsection{Method validation}

Institutional review board approval was obtained for the use of human plasma samples, according to the Declaration of Helsinki. Thirty plasma samples obtained from Rennes University Hospital patients between February 2010 and February 2013 were analysed using RIA method. Residual plasma samples were re-analysed by LCHRMS method, and results were compared to RIA values. Blood was collected in EDTA tubes, and plasma was separated by centrifugation at $3000 \mathrm{~g}$ for 10 minutes. Then, plasma was aspirated gently with respecting a security merge of $1 \mathrm{~cm}$ from the buffy coat to avoid contamination by white blood cells, and was frozen at $-80^{\circ} \mathrm{C}$ until assayed. Samples have been stored at $-80^{\circ} \mathrm{C}$ until analysis and treated by the same number of freeze-thaw cycles. HA quantification by RIA in plasma was realised using RIA Histamine kit (Immunotech, Marseilles, France) according to the manufacturer's procedure.

For HA quantification using the in-house LCHRMS method, plasma samples were prepared as QC: $100 \mu \mathrm{L}$ of plasma sample were added to $250 \mu \mathrm{L}$ of de-ionized water and $100 \mu \mathrm{L}$ of IS. Sulfosalicylic acid (SSA) $(50 \mu \mathrm{L}$ at $20 \% \mathrm{v} / \mathrm{v})$ was added in each tube for protein precipitation before vortexing for 30 seconds. After centrifugation at $3000 \mathrm{~g}$ for 10 minutes at $4^{\circ} \mathrm{C}, 200 \mu \mathrm{L}$ of supernatant were transferred to an auto-sample vial.

\subsection{Statistical analysis}

GraphPad Prism 5.0 (GraphPad Software, San Diego, CA, USA) and Excel software were used to perform statistical analyses. LCHRMS and RIA were compared using a Spearman correlation analysis. Results are expressed as means \pm SD. A p-value of less than 5\% was considered statistically significant.

\section{Results}

During method development, different procedures have been evaluated to optimize sample extraction, matrix effect, chromatographic and detection parameters. Sample preparation, extraction and chromatographic conditions have been carefully optimized for simple, rapid and practical quantitative analyse, avoiding most of matrix effects.

\subsection{Chromatography and detection}


Mass spectra showing the selected precursor and product ion used for detection of HA and deuterated Histamine (HAd4) are presented in Figure 1. The precursor ion of HA displays an $\mathrm{m} / \mathrm{z}$ of 112.0871 which is an error of $1.57 \mathrm{ppm}$ with the theoretical mass assigned by X-Calibur, ([Observed Ion Mass - Theoretical Ion Mass] / Theoretical Ion Mass) x 106 . Its primary product ion has an $\mathrm{m} / \mathrm{z}$ of 95.0607 . The precursor ion of HAd4 shows an $\mathrm{m} / \mathrm{z}$ of 116.1120, and its primary product ion an $\mathrm{m} / \mathrm{z}$ of 99.0855 . Representative LCHRMS chromatograms of samples are shown in Figure 2. Each chromatogram represented the two modes of acquisition in full scan and targeted MS2. The first mode allows the quantification and the second confirms specific detection. HA and HAd4 have the same retention time of $4.1 \mathrm{~min}$ (Figure 2). We hypothesize that the small peak at 4.3 min corresponds to a stereoisomer.

Chromatograms of protonated histamine (HA) and HAd4, obtained by full scan acquisition and targeted MS2 mode using a 5 ppm mass window for a human plasma sample (approximatively $20 \mathrm{nM}$ )

\subsection{Calibration curve and linearity}

The six-point calibration curve for histamine was linear over the range of 1 to $180 \mathrm{nM}$ with CVs ranging from 1.6 to $8.1 \%$. The linearity was verified with a lack of fit test and the $\mathrm{r}^{2}$ coefficient of determination was $>0.99$. Typical equation of calibration curve was: $y=0.9902 x$ +0.9735 . The LLOQ was determined to be $1 \mathrm{nM}(\mathrm{CV}=8.1 \%)$.

\subsection{Recovery, process efficiency and matrix effect}

RE was determined between 102.1 and $114.9 \%$. In this procedure, ME was determined to be lower than $3 \%$. Also, PE has been calculated at $+11.4 \%,+7.8 \%$ and $-0.5 \%$ respectively for 3 , 30 and $150 \mathrm{nM}$ QC samples.

\subsection{Precision and Accuracy}

Six aliquots of each QC samples $(3,30,150 \mathrm{nM})$ were tested in the same run to evaluate repeatability. Mean, $\mathrm{SD}, \mathrm{CVs}$, and biases are reported to be acceptable (table 2). The inter-day evaluation of QC samples tested once a day $(n=10)$ showed a good reproducibility (Table 3). All CVs were inferior to $10 \%$ for the 3 levels $(8.7,6.1$ and $3.4 \%$ for 3,30 and $150 \mathrm{nM}$ QC samples, respectively) and biases were between 0.2 and $6.1 \%$. The precision and accuracy were in conformity with the related rules of biological sample analysis method guidelines $[20]$. 


\subsection{Stability}

Using our validated method, we assessed the stability of HA under various conditions. Concerning the stability of stock solutions at $-80^{\circ} \mathrm{C}$, we did not observe any decrease in HA concentration after 1 year of storage. The stability of QC samples at $-80^{\circ} \mathrm{C}$ was also excellent for the three QC samples. For instance, 8 months after preparation and with a new calibration solution, the 3, 30 and $150 \mathrm{nM}$ QC samples showed measured values of 3.00, 30.09, and $161.4 \mathrm{nM}$, respectively. In addition, mean concentrations over 8 months were $2.99(\mathrm{CV}=$ $3.46 \%), 31.02(\mathrm{CV}=2.85 \%)$ and $157.84 \mathrm{nM}(\mathrm{CV}=2.90 \%)$. Finally, HA stability after extraction also showed that no significant degradation occurred during auto-sampler at $15^{\circ} \mathrm{C}$ for $12 \mathrm{~h}$ before analysis. A decrease of 7.3, 2.5 and 5.5\% was observed for the 3 QC samples (3, 30 and $150 \mathrm{nM}$, respectively).

\subsection{Plasma sample analysis and comparison with RIA}

HA concentration of plasma samples from patients has been evaluated using our validated LCHRMS method, and compared to the concentration obtained with the gold standard RIA method. Among the 30 plasma samples tested, only 25 had a HA concentration comprised within the limit of quantification of the RIA method, as defined by the manufacturer ( $1 \mathrm{nM}$ to $100 \mathrm{nM}$ ). For these samples, an excellent correlation was revealed for HA concentrations determined by the two methods $\left(r^{2}=0.9198\right.$, Figure 3$)$. Concerning the 5 remaining samples, HA concentration of 3 of them was measured superior to $100 \mathrm{nM}$ using RIA and LCHRMS, and the 2 other samples were evaluated to have a HA concentration inferior to $1 \mathrm{nM}$ with both methods.

\section{Discussion}

HA quantification is useful to confirm that clinical signs of a suspected anaphylaxis reaction result from the degranulation of tissue mast cells and blood basophils. This confirmation leads to a clinical and/or biological allergic investigation in order to find the offending allergen against which the patient is sensitized, allowing thereafter avoiding a new anaphylaxis reaction by the specific eviction of the allergen, or an induction of tolerance. HA quantification with the RIA gold standard method has the advantage to be highly sensitive, but requires the use of radioactive elements that implies the formation and the follow-up of the technicians, as well as the radioactive waste management, which is dangerous and expensive. Therefore, the 
development of new methods presenting fewer disadvantages seems necessary. Methods using mass spectrometry are now commonly used in medical laboratories for quantification of drugs or metabolites. Recently, Liu J. et al described a UHPLC-MS/MS method for HA quantification in plasma of four different mammalian species including humans [21]. This method presents important advantages: low time-consuming, simple method. Unfortunately, blood samples were collected into tubes containing heparin sodium. However, it has been well-documented that heparin could bind to HA [22]; this phenomenon could then modify the free HA concentration measured by MS in the samples. The sensitivity of the method for HA quantification seems to be lower than for other methods classically used, due to a very low signal-to-noise ratio at the LLOQ value. In addition, a statistical correlation with obtained concentrations using RIA kits had not been determined. Furthermore, five plasma samples from healthy volunteers have been tested with this method. Plasma histamine amount is known to be physiologically very low in healthy patients. Nevertheless, in the reported study, two out of these five samples showed a HA concentration superior to $10 \mathrm{nM}(12.7 \mathrm{nM}$ and 24.7 $\mathrm{nM}$ ), concentration classically considered as the positive threshold value determining an HA release after mast cell and/or basophil degranulation. More recently, another LCMSMS method for HA quantification in rat plasma has been described by Chimalakonda K. et al [23]. With this method, the LLOQ was determined at $156 \mathrm{nM}$. However, this value is 15 fold higher than the positive threshold usually used for clinical diagnosis. Overall, these technically advantageous methods require some complementary experiments before being recommended in medical laboratories for a diagnostic use.

In our study, High Resolution Accurate Mass LC-MS technology was chosen for HA quantification due to its high specificity and sensitivity for low molecular weight molecule quantification. Due to low amounts of HA physiologically found in plasma of healthy people, TrisBSA was used to mimic plasma HA-free matrix. Three QC samples were prepared by fortifying TrisBSA with HA: low at $3 \mathrm{nM} \mathrm{HA}$, weakly positive at $30 \mathrm{nM} \mathrm{HA}$, and highly positive at $150 \mathrm{nM}$ HA. Recovery, matrix effect and process efficiency have also been tested in plasmas fortified with 3, 30 and $150 \mathrm{nM}$ of HA. Process efficiency was similar between fortified plasma and TrisBSA (data not shown). Because HA concentrations over $100 \mathrm{nM}$ reflect a massive mast cell and/or basophil degranulation and have no diagnostic interest, this value was chosen as the upper LOQ. The lower LOQ was determined at $1 \mathrm{nM}$, which is classically reported with other methods. For any steps of experiments, plastic containers were used, and glass surfaces were forbidden in order to avoid HA absorption, as described by Verburg and Henry [24]. Considering the extraction procedure, protein precipitation was performed using SSA. The mobile phase combined an increasing NFPA rate with an increasing flow rate in $5 \mathrm{~min}$. Volatile ion-pair reagents 
with long alkyl chain as NFPA are known to improve retention on ionisable polar compounds and to diminish matrix effect [25]. Moreover, in 1996, Pearson et al showed that the use of longer alkyl chained perfluorinated carboxylic acids may be an option to solve such problems [26]. The first 4 min were necessary to elute HA and the IS. The total run time of the method was set to $10 \mathrm{~min}$ in order to eliminate all interferences and re-equilibrate the column for the next injection. Between 2 injections, a $3 \mathrm{~mL}$ flush $(75 \% \mathrm{ACN}, 25 \%$ water containing $0.1 \%$ TFA) was necessary to minimized carryover.

Simplification of sample preparation steps is crucial for a routinely medical laboratory use. However, most of HA quantification methods used for medical applications needs a derivatization step [7][14], leading to an increased risk of low analyte recovery, and extended sample preparation and analysis time. With High Resolution Accurate Mass LC-MS technology, derivatization, solid phase or extraction are not necessary. In addition, a limited volume of $100 \mu \mathrm{L}$ of plasma sample is sufficient to obtain a good sensitivity.

Finally, the comparison of HA quantification using LCHRMS and RIA in plasma samples showed a very good correlation reinforcing the possibility to use our LCHRMS method in medical laboratory. Although results found by LCHRMS are well correlated with results obtained by RIA, some results are slightly underestimated with LCHRMS particularly for concentrations above $30 \mathrm{nM}$. We can discuss some hypotheses to explain these discrepancies. Firstly, because plasma samples have been analysed by RIA in 2010 and by LCHRMS three years later, we could hypothesize that the underestimation obtained with LCHRMS could be explained by a possible degradation of HA during the prolonged storage at $-80^{\circ} \mathrm{C}$. However, Laroche et al. reported that HA is stable in plasma for a period of at least 3 years at $-20^{\circ} \mathrm{C}$ [27]. Secondly, according to the manufacturers' data, cross-reactivity with methyl histamine, the main metabolite of HA, is estimated at $0.069 \%$ with RIA. LCHRMS is the only assay that can distinguish methyl histamine from HA. Thirdly, LCHRMS calibration curve is linear at least from 1 to $240 \mathrm{nM}$ (data not shown), whereas RIA calibration curve is a semi-logarithmic. Considering samples with HA concentrations comprised between 30 and $100 \mathrm{nM}$, dots are situated at the plateau of the calibration curve (see graph below), which probably induces less precision and accuracy. Further investigations are underway to explore this underestimation with the LCHRMS method. Nevertheless, LCHRMS presents the advantage to avoid use of radioactivity. And even with a run time of 10 min per sample for a good metabolite separation, LCHRMS method remains still faster than RIA. 


\section{Conclusion}

In summary, we validated an original and specific LC-High Resolution-MS method for HA quantification in plasma samples. The $\mathrm{Q}$ Exactive $^{\mathrm{TM}}$ mass spectrometer was demonstrated to be suitable for routinely quantification in medical laboratories, and was less time-consuming than RIA, while exhibiting a similar sensitivity without derivatization step. We validated this LC-High Resolution-MS method for all the following parameters: linearity, matrix effect, precision, recovery and stability. In addition, this method was successfully applied to small plasma volumes.

Overall, we report here a new method for histamine quantification in biological human plasmas using LCHRMS, suitable for routinely use in medical laboratories. Interestingly, LC-MS consumables are clearly safer and cheaper than those used for RIA. In addition, medical laboratories will try in a near future to avoid radioactivity use for safety issue. Then, despite the expensiveness of the device, we validate that LCHRMS could be a good candidate to replace RIA as the Gold Standard for HA quantification. 


\section{Acknowledgments}

The authors thanks the "Fonds d'Innovation Interne" of the University Hospital of Rennes for its financial support 


\section{Conflict of interest}

The authors declare no conflict of interest. 


\section{REFENCES}

[1] Dale HH, Laidlaw PP. The physiological action of $\beta$-iminazolylethylamine. J Physiol 1910;41:318-44.

[2] Croyal M, Dauvilliers Y, Labeeuw O, Capet M, Schwartz J-C, Robert P. Histamine and tele-methylhistamine quantification in cerebrospinal fluid from narcoleptic subjects by liquid chromatography tandem mass spectrometry with precolumn derivatization. Anal Biochem 2011;409:28-36. doi:10.1016/j.ab.2010.09.045.

[3] Rosa A c., Fantozzi R. Histamine in the neurogenic inflammation. Br J Pharmacol 2013:n/a - n/a. doi:10.1111/bph.12266.

[4] Brew O, Sullivan MHF. The links between maternal histamine levels and complications of human pregnancy. J Reprod Immunol 2006;72:94-107. doi:10.1016/j.jri.2006.04.002.

[5] Maintz L, Schwarzer V, Bieber T, van der Ven K, Novak N. Effects of histamine and diamine oxidase activities on pregnancy: a critical review. Hum Reprod Update 2008;14:485-95. doi:10.1093/humupd/dmn014.

[6] Hogan A-M, Crean C, Barrett UM, Guihen E, Glennon JD. Histamine determination in human urine using sub-2 $\mu \mathrm{m} \mathrm{C18}$ column with fluorescence and mass spectrometric detection. J Sep Sci 2012;35:1087-93. doi:10.1002/jssc.201101045.

[7] Wang Z, Wu J, Wu S, Bao A. High-performance liquid chromatographic determination of histamine in biological samples: The cerebrospinal fluid challenge - A review. Anal Chim Acta 2013;774:1-10. doi:10.1016/j.aca.2012.12.041.

[8] Koyama J, Takeuchi A, Tode C, Shimizu M, Morita I, Nobukawa M, et al. Development of an LC-ESI-MS/MS method for the determination of histamine: application to the quantitative measurement of histamine degranulation by KU812 cells. J Chromatogr B Analyt Technol Biomed Life Sci 2009;877:207-12. doi:10.1016/j.jchromb.2008.12.012.

[9] Zhang Y, Tingley III FD, Tseng E, Tella M, Yang X, Groeber E, et al. Development and validation of a sample stabilization strategy and a UPLC-MS/MS method for the simultaneous quantitation of acetylcholine (ACh), histamine (HA), and its metabolites in rat cerebrospinal fluid (CSF). J Chromatogr B 2011;879:2023-33. doi:10.1016/j.jchromb.2011.05.030.

[10] García-Villar N, Hernández-Cassou S, Saurina J. Determination of biogenic amines in wines by pre-column derivatization and high-performance liquid chromatography coupled to mass spectrometry. J Chromatogr A 2009;1216:6387-93. doi:10.1016/j.chroma.2009.07.029.

[11] Cunha SC, Faria MA, Fernandes JO. Gas chromatography-mass spectrometry assessment of amines in Port wine and grape juice after fast chloroformate extraction/derivatization. J Agric Food Chem 2011;59:8742-53. doi:10.1021/jf201379x.

[12] Pittertschatscher K, Hochreiter R, Thalhamer J, Hammerl P. Quantification of histamine in blood plasma and cell culture supernatants: a validated one-step gas chromatography-mass spectrometry method. Anal Biochem 2002;308:300-6. doi:10.1016/S00032697(02)00260-9.

[13] Nishiwaki F, Kuroda K, Inoue Y, Endo G. Determination of histamine, 1-methylhistamine and N-methylhistamine by capillary electrophoresis with micelles. Biomed Chromatogr BMC 2000;14:184-7. doi:10.1002/1099-0801(200005)14:3<184::AIDBMC970>3.0.CO;2-2.

[14] Morel AM, Delaage MA. Immunoanalysis of histamine through a novel chemical derivatization. J Allergy Clin Immunol 1988;82:646-54.

[15] McBride P, Bradley D, Kaliner M. Evaluation of a radioimmunoassay for histamine measurement in biologic fluids. J Allergy Clin Immunol 1988;82:638-46.

[16] Guesdon JL, Chevrier D, Mazié JC, David B, Avrameas S. Monoclonal anti-histamine antibody. Preparation, characterization and application to enzyme immunoassay of histamine. J Immunol Methods 1986;87:69-78. 
[17] Fujiwara K, Murata I, Yagisawa S, Tanabe T, Yabuuchi M, Sakakibara R, et al. Glutaraldehyde (GA)-hapten adducts, but without a carrier protein, for use in a specificity study on an antibody against a GA-conjugated hapten compound: histamine monoclonal antibody (AHA-2) as a model. J Biochem (Tokyo) 1999;126:1170-4.

[18] Matuszewski BK, Constanzer ML, Chavez-Eng CM. Strategies for the Assessment of Matrix Effect in Quantitative Bioanalytical Methods Based on HPLC-MS/MS. Anal Chem 2003;75:3019-30. doi:10.1021/ac020361s.

[19] Causon R. Validation of chromatographic methods in biomedical analysis. Viewpoint and discussion. J Chromatogr B Biomed Sci App 1997;689:175-80.

[20] U.S. Department of Health and Human Services, Food and Drug Administration, Center for Drug Evaluation and Research. Guidance for Industry, Bioanalytical Method Validation. 2008. Guid Ind Bioanal Method Valid 20082001.

[21] Liu J, Wang L, Hu W, Chen X, Zhong D. Development of a UHPLC-MS/MS method for the determination of plasma histamine in various mammalian species. J Chromatogr B Analyt Technol Biomed Life Sci 2014;971:35-42. doi:10.1016/j.jchromb.2014.08.043.

[22] Rabenstein DL, Bratt P, Peng J. Quantitative characterization of the binding of histamine by heparin. Biochemistry (Mosc) 1998;37:14121-7. doi:10.1021/bi980625y.

[23] Chimalakonda KC, Pang E, Weaver JL, Howard KE, Patel V, Boyne MT. Development and validation of a liquid-chromatography tandem mass spectrometry method to determine in vitro and in vivo histamine release. J Pharm Biomed Anal 2015;102:494-9. doi:10.1016/j.jpba.2014.10.016.

[24] Verburg KM, Henry DP. Binding of histamine by glass surfaces. Agents Actions 1984;14:633-6.

[25] Gao S, Bhoopathy S, Zhang Z-P, Wright DS, Jenkins R, Karnes HT. Evaluation of volatile ion-pair reagents for the liquid chromatography-mass spectrometry analysis of polar compounds and its application to the determination of methadone in human plasma. $\mathrm{J}$ Pharm Biomed Anal 2006;40:679-88. doi:10.1016/j.jpba.2005.05.022.

[26] Pearson JD, McCroskey MC. Perfluorinated acid alternatives to trifluoroacetic acid for reversed-phase high-performance liquid chromatography. J Chromatogr A 1996;746:27781.

[27] Laroche D, Dubois F, Gérard J-L, Lefrançois C, André B, Vergnaud M-C, et al. Radioimmunoassay for plasma histamine: a study of false positive and false negative values. Br J Anaesth 1995;74:430-7. doi:10.1093/bja/74.4.430. 


\section{TABLES}

\begin{tabular}{cccc} 
Time (minute) & Solvent A $(\%)$ & Solvent B $(\%)$ & Flow rate $\left(\mu \mathrm{L} \cdot \mathrm{min}^{-1}\right)$ \\
\hline 0 & 10 & 90 & 400 \\
3 & 13 & 87 & 600 \\
5 & 53 & 47 & 600 \\
5.50 & 10 & 90 & 600 \\
7 & 10 & 90 & 400 \\
\hline
\end{tabular}

Table 1: Liquid chromatography gradient elution. Solvent A: NFPA (3 mmol.L $\left.{ }^{-1}\right)$. Solvent B: Acetonitrile with $0.1 \%(v / v)$ formic acid. 


\begin{tabular}{lccc}
\hline & Low $(\mathrm{QC} 3)$ & Middle (QC30) & High (QC150) \\
\hline Theoretical concentration (nM) & 3.0 & 30.0 & 150.0 \\
Measured concentration (nM) & 2.9 & 30.7 & 151.4 \\
SD (nM) & 0.1 & 1.3 & 4.4 \\
Intra-day CV (\%) & 2.3 & 4.1 & 2.9 \\
Bias (\%) & 4.1 & 2.4 & 0.9 \\
\hline
\end{tabular}

Table 2: Intra-day precision and bias for 3 levels of histamine QC samples (data are mean $\pm S D, n=6)$. ( $S D=$ Standard deviation, $C V=$ coefficient of variation $)$ 


\begin{tabular}{lccc}
\hline & Low (QC3) & Middle (QC30) & High (QC150) \\
\hline Theoretical concentration (nM) & 3.0 & 30.0 & 150.0 \\
Measured concentration $(\mathrm{nM})$ & 3.0 & 31.7 & 159.1 \\
SD (nM) & 0.3 & 1.9 & 5.4 \\
Inter-day CV (\%) & 8.7 & 6.1 & 3.4 \\
Bias (\%) & 0.2 & 5.6 & 6.1 \\
\hline
\end{tabular}

Table 3: Inter-day precision and bias for 3 levels of histamine QC samples (data are mean $\pm S D, n=10) .(S D=$ Standard deviation, $C V=$ coefficient of variation $)$ 


\section{FIGURES}
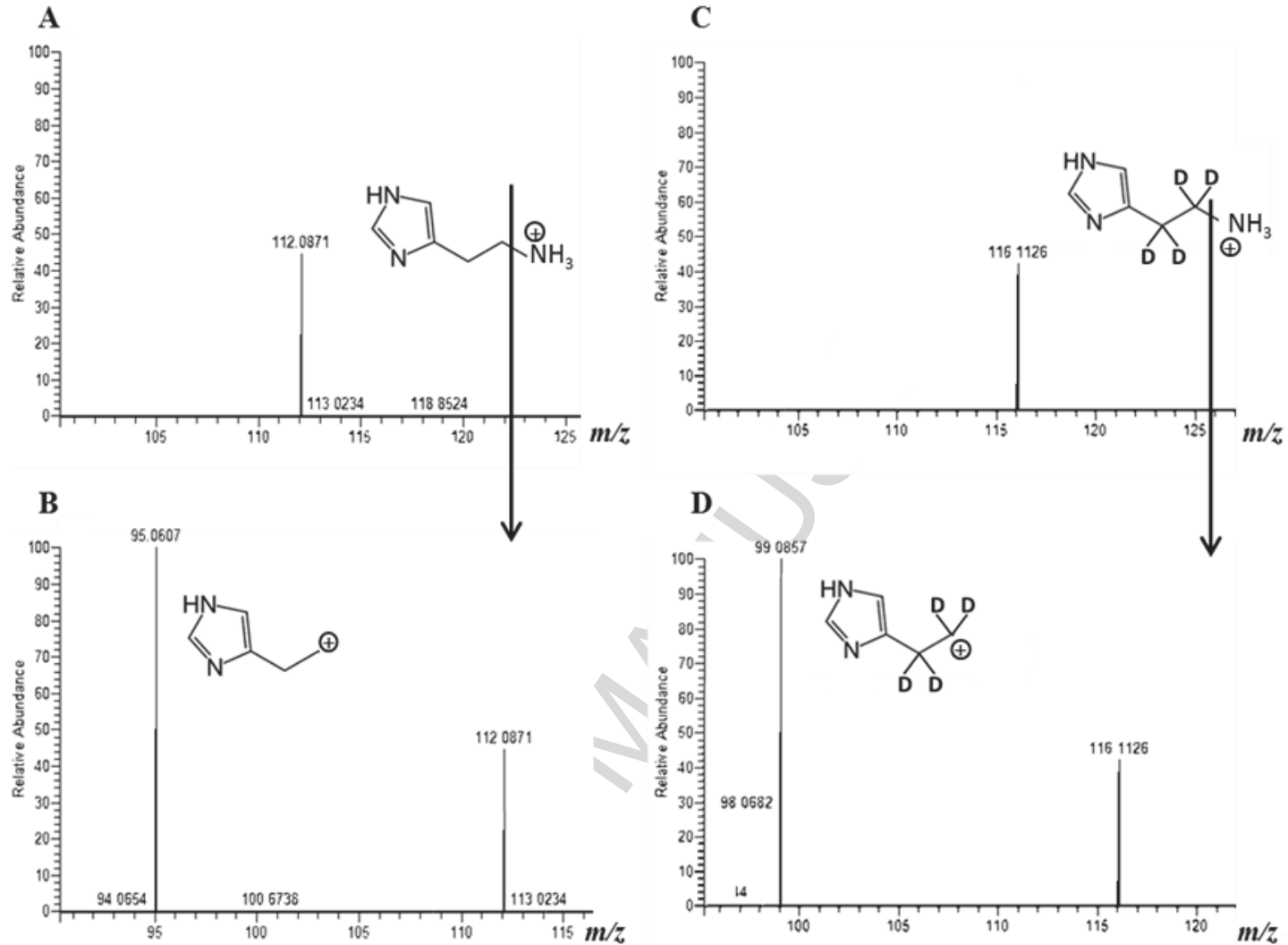

Figure 1: Accurate mass spectra of the precursor and product ions of HA and HAd4. (A) The Molecular ion of protonated HA; (B) Majors fragments of HA; (C) Molecular ion of protonated HAd4; (D) Majors fragments of HAd4 


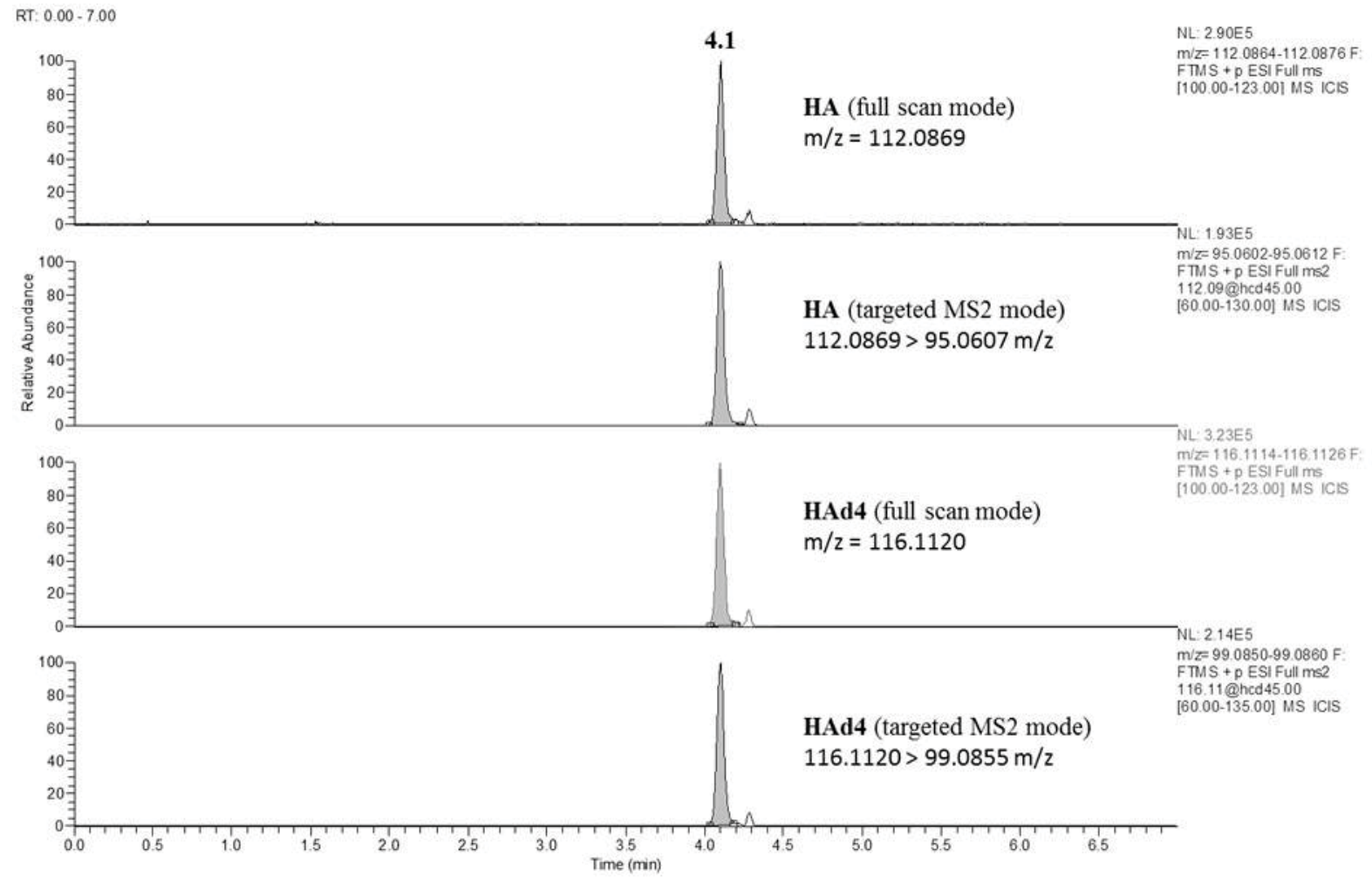

Figure 2: Representative chromatograms obtained from human plasma. 


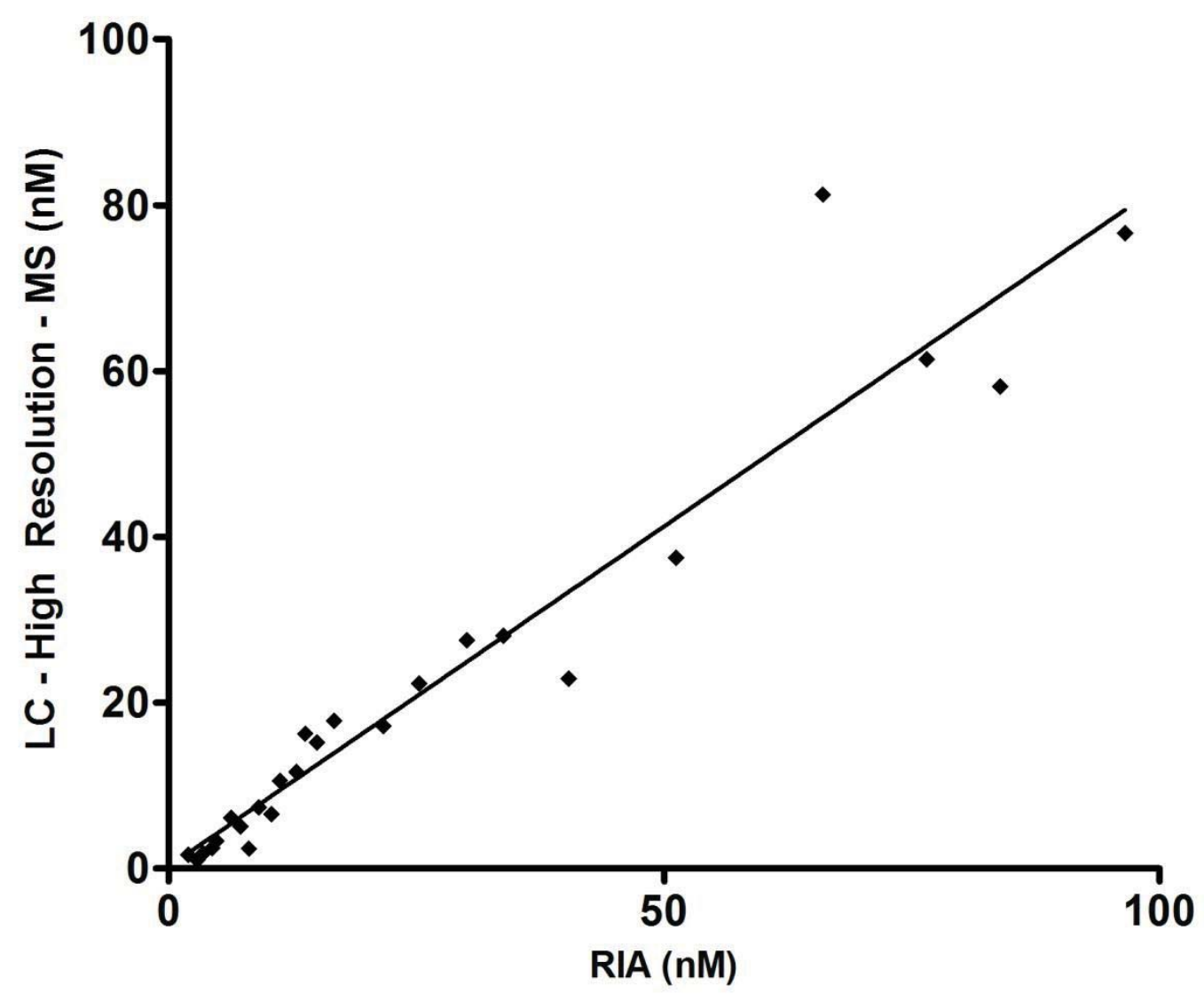

Figure 3: Comparison of HA concentrations obtained by LC-High Resolution-MS analysis versus RIA. HA concentration of 25 plasma samples obtained from patients was measured using LCHRMS and RIA. 


\section{Highlights}

Q-Exactive mass spectrometer is suitable for routinely use in medical laboratories

An LCHRMS method for histamine quantification has been developed for human plasma

The LCHRMS method shows a good correlation with the gold standard RIA assay 